Classification

Physics Abstracts

$61.50 \mathrm{~J}-63.90$

\title{
Fractal structure of the equilibrium crystal shape. II. Fractal dimensions
}

\author{
S. E. Burkov \\ Landau Institute for Theoretical Physics, U.S.S.R. Academy of Sciences, \\ Kosygina 2, 117940, GSP-1, Moscow V-334, U.S.S.R.
}

(Reçu le 12 mars 1985, accepté le 10 juillet 1985)

\begin{abstract}
Résumé. - La forme d'un cristal à $T=0$ est étudiée dans un modèle de " briques » s'attirant selon une loi en $1 / r^{\gamma}$. On démontre que les côtés et les sommets isolés sont des ensembles de Cantor de dimension fractale $\gamma /(\gamma-2)$ et $6 /(\gamma-1)$, respectivement.
\end{abstract}

\begin{abstract}
A crystal shape at $T=0$ is studied in the framework of the model of « bricks " attracting each other according to $1 / r^{\gamma}$. It is shown that fractal dimensions of the Cantor sets of solitary edges and corners are $\gamma /(\gamma-2)$ and $6 /(\gamma-1)$, respectively.
\end{abstract}

Recently equilibrium crystallization phenomena have been extensively studied. In some experiments a crystal $\left(\mathrm{He}^{4}[1,2]\right.$ or $\left.\mathrm{Pb}[3]\right)$ has time to achieve the thermodynamic equilibrium shape. Before investigating the thermodynamics it is useful to calculate a crystal shape at $T=0$. When thermal and quantum fluctuations are neglected the equilibrium crystal shape (ECS) is a shape of absolutely minimal surface energy, provided the volume $V$ is fixed. Naturally, we are interested in the limit $V \rightarrow \infty$, so the function $z(x, y ; V)$, describing the surface of a crystal of the volume $V$, must be replaced by a function $Z(X, Y)=\lim _{V \rightarrow \infty} z\left(V^{1 / 3} X, V^{1 / 3} Y ; V\right) / V^{1 / 3}$. This function describes the sizes of the facets and their orientations but not the atomic structure of the interface, the facets becoming plane polygons. The function $Z(X, Y)$ can be differentiated only once. So, the unit vector $\mathbf{n}$ normal to the crystal surface takes all possible values and varies continuously (i.e. there is no sharp edge or slope discontinuity). Nevertheless, the crystal is completely faceted (i.e. there is no rounded area). The surface consists of an infinite number of facets (the larger the Miller index, the smaller the facet) and of two types of Cantor sets.

It is useful to deal with these sets in the parametric representation. The crystal shape will be described by three functions $X, Y, Z$ of two parameters instead of the function $Z(X, Y)$. A unit normal vector $\mathbf{n}$ will be chosen as those two parameters, making it possible to use the Wulff construction [4]. If both parameters are irrational a single point or solitary corner appears on the crystal surface. All these points form a Cantor set of the first type. If one component of $\mathbf{n}$ is rational but the other one is irrational, a straight line interval or " solitary edge " appears on the surface. All these intervals form a Cantor set of the second type. If both slopes are rational there is a facet (polygon) in the ECS. Along with these two " surface " Cantor sets there are " linear " Cantor sets in the boundaries of every facet. Namely, a contour confining the facet can be parametrized 
by one slope. Rational slopes give rise to edges, irrational slopes give rise to single points (corners) in the curve. All points of irrational slope form a Cantor set. (Exact definitions, statements and details can be found in Ref. [5], which will be cited below as I.)

There is a model, first introduced by Landau [6], which allows one to calculate the ECS $Z(X, Y)$ exactly. It can be called a " model of attracting bricks ». The atoms are supposed to be cubic bricks forming a simple cubic lattice (the lattice constant is put equal to unity). The lattice is assumed to be undeformable, so the centres of the bricks occupy integer points only. The interaction between each two atoms is given by the potential $U(\mathbf{r})$. It must decrease at large distances more rapidly than $r^{-4}$ and must be negative : $U(r)<0$ (see Fig. 1 of I). The second condition is necessary. If it does not hold the ECS does not possess the analytic properties mentioned above (sharp edges emerge instead of Cantor sets). Although the shapes of all facets have been found exactly in the framework of this model, here we apply the results of I to high index facets only. There are two reasons to do so. First, only the smallest facets (they have large indices) are relevant for fractals. Second, the brick model itself properly describes only small facets of a real crystal. Moreover, the accuracy of this " brick approximation " improves as a facet size tends to zero. For this reason we hope that the brick model yields exact values of fractal dimensions. To investigate high index facets it is necessary to know $U(\mathbf{r})$ at large $r$ only (because small $r$ are cancelled). Bearing in mind the Van der Waals attraction we put $U(\mathbf{r})=-1 / r^{\gamma}$ at $r \gg 1$. The aim of this paper is to report that the fractal dimensions of the Cantor sets are : $\frac{6}{\gamma-1}$ for solitary corners, $\frac{\gamma}{\gamma-2}$ for solitary edges, and $\frac{2}{\gamma-1}$ for « linear » Cantor sets in facet boundaries.

\section{Facet boundary.}

We fix some indices $\{p, q, r\}$ and introduce a vector $\mathbf{N}=(p, q, r)$ normal to the facet (Fig. 7 of $\mathrm{I}, \mathbf{n}=\mathbf{N} / N)$. The contour which confines this facet consists of an infinite number of intervals (edges) and of a Cantor set of single points (it is similar to the curve shown in Fig. 6 of I). To calculate the fractal dimension of this set one must choose a small scale $\Delta$ and consider the curve with the accuracy up to $\Delta$. Namely, we must plot all intervals longer than $\Delta$ (smaller edges are undiscernible at given resolution $\Delta$ ). The curve consists of $M$ longest intervals and of $M$ gaps between them (Fig. 6 of I). The gaps contain edges, shorter than $\Delta$, and the Cantor set. The length of an edge is

$$
\Delta L=-\sum_{m=1}^{\infty} m l U(m l)=\frac{\zeta(\gamma-1)}{l^{\gamma-1}}
$$

where $l$ is the smallest integer vector parallel to the edge. So, we must consider all integer vectors 1 belonging to the plane $\mathbf{N} . \mathbf{l}=0$ and shorter than some large $\Lambda ;\left(\Delta=\zeta(\gamma-1) / \Lambda^{\gamma-1}\right)$. The number of these vectors is known from elementary number theory [7] :

$$
M=\frac{\pi}{\zeta(2) N} \Lambda^{2}=\frac{6}{\pi N} \Lambda^{2}
$$

Finally, we must estimate the widths of the gaps. It can be done making use of the formulae of I and all the gaps are of the order of $\Delta$. Substituting $\Lambda \sim \Delta^{-\frac{1}{\gamma-1}}$ into equation (2) we have for large $M$ :

$$
M \sim \Delta^{-d}, \quad d=\frac{2}{\gamma-1} .
$$


So, the fractal dimension is $2 /(\gamma-1)$. Note that the number of the discernible edges increases more slowly than $\Delta^{-1}$ as one improves the resolution. This is due to the presence of the "Cantor dust » in the gaps.

\section{Solitary edges.}

As has been mentioned above, one slope of a solitary edge is rational but the other is irrational. One can construct a subset of the set of all the solitary edges fixing the rational slope when the second parameter takes all irrational values. Along with this Cantor set one can construct a oneparameter subset of the crystal surface if one assumes that the second slope takes all possibles values (both rational and irrational). This subset contains an initial Cantor set and an infinite number of facets. It resembles a setting embracing the crystal (Fig. 1). More accurately, the setting must be constructed as follows : we fix some integer vector $\mathbf{l}$ and consider only those unit vectors $\mathbf{n}$ which belong to the plane $\mathbf{n . l}=0$. Remembering that two independent components of a unit vector $\mathbf{n}$ normal to the crystal surface are considered as two parameters, we conclude that if $\mathbf{n}$ is in the plane $\mathbf{n} . \mathbf{l}=\mathbf{0}$ a one-parameter subset appears on the crystal surface. The boundary of every facet of the setting contains two edges parallel to $\mathbf{l}$. Their length $\Delta L$, given by equation (1), is index-independent, so all these edges (belonging to different facets of the same setting) have the same length $\Delta L$. Note that the length of the solitary edges is also $\Delta L$. Consider high index facets of given setting. They are very narrow : their lengths in the direction of 1 almost equal $\Delta L$, but their widths $\Delta R(\mathbf{N})$ in the plane perpendicular to $\mathbf{l}$ tend to zero as $N=\sqrt{p^{2}+q^{2}+r^{2}} ;(\mathbf{n}=\mathbf{N} / N$, $\mathbf{N} . \mathbf{l}=0)$ increases. The widths $\Delta R(\mathbf{N})$ can be found from equation $(21)$ of $\mathrm{l}$, where $\mathrm{e}=(\mathbf{N} \times \mathbf{l}) / N l$ that formula contains the summation over 2-D «Diophantine " lattice. Two basic vectors of that lattice must be chosen so that $I$ is one of them. (Eq. (22) of I is not convenient for the particular problem.) For large $N$ the summation in equation (21) of I can be performed :

$$
\Delta R(\mathbf{N})=\varphi(\gamma) \zeta(\gamma-2) l^{\gamma-3} / N^{\gamma-2}
$$

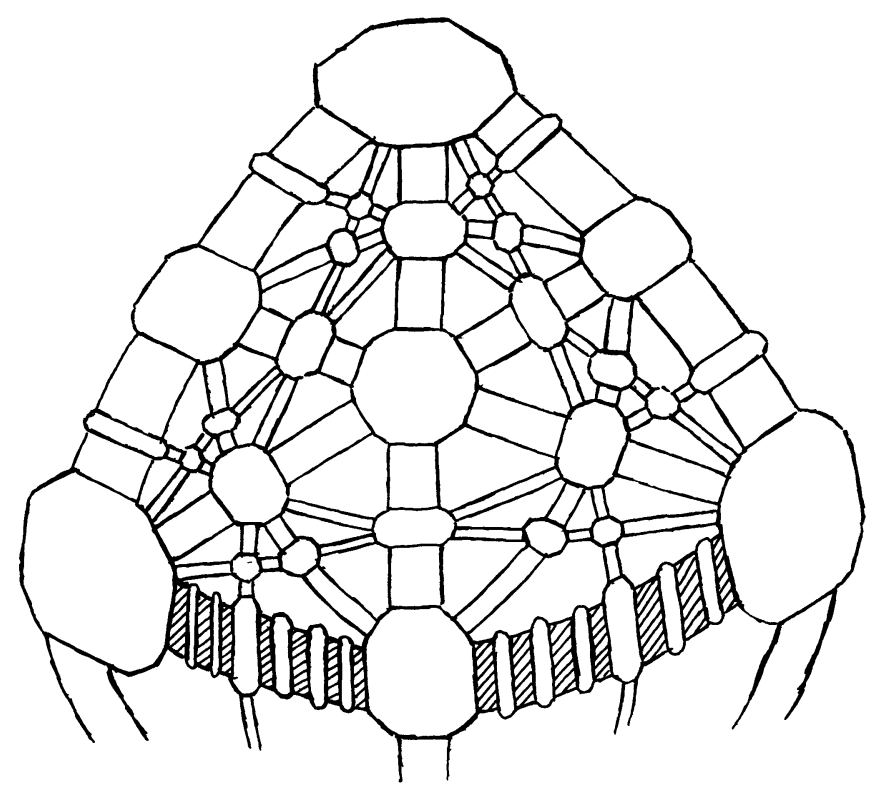

Fig. 1. - Schematic plot of the ECS. A one-parameter subset with $\mathbf{l}=(0,0,1)$ (a horizontal setting) is shown with higher resolution. The gaps containing the solitary edges parallel to the $Z$-axis are shaded. 
where $\varphi(\gamma)=\mu(\gamma)(\gamma-3) ! ! /(\gamma-2) ! ! ; \mu(\gamma)=2$ if $\gamma$ is odd, $\mu(\gamma)=\pi$ if $\gamma$ is even. In other words, the setting is something like a cylinder with the generatrix $\Delta L$ and with the base bounded by a contour analogous to the one shown in figure 6 of $I$.

It is useful to calculate the fractal dimension of the Cantor set of the solitary edges of one setting only. So we fix some integer $\mathbf{l}$ and the corresponding one-parameter subset on the crystal surface. Then we fix a small scale $\Delta$. We choose $\Delta \ll \Delta L$ and, since the longitudinal (parallel to l) size of the setting $\Delta L$ is constant, we can deal with transversal sizes only. Then we must construct all facets of the width larger than $\Delta$ (high index facets which are narrower than $\Delta$ are undiscernible at given resolution $\Delta$ ). The setting consists of $M$ widest facets and of $M$ gaps. Every gap is a parallelogram, two long sides being of the length $\Delta L(\mathbf{l})$ whereas the transversal size is of the order of $\Delta$. The gaps contain the facets narrower than $\Delta$ and the Cantor set of solitary edges. The facet width is given by equation (4), so we must consider all the integer vectors $\mathbf{N}$ lying in the plane $\mathbf{N} .1=0$ which are shorter than some large $\Lambda$. The number of these vectors is given by a formula analogous to equation (2) $: M=6 \Lambda^{2} / \pi l$. Substituting $N \sim \Lambda$ into equation (4) we have $\Delta \sim 1$ / $\Lambda^{\gamma-2}$ and finally :

$$
M \sim \Delta^{-\frac{2}{\gamma-2}}
$$

giving the number of discernible facets as the function of the resolution $\Delta$ (Fig. 2a). Bearing in mind that we deal with the solitary edges (intervals) the fractal dimension is $\frac{2}{\gamma-2}+1=\frac{\gamma}{\gamma-2}$. It does not depend on $\mathbf{l}$, so the fractal dimension of the set of all solitary edges (with all orientations) is also $\gamma /(\gamma-2)$.

\section{Solitary corners.}

A solitary corner is a single point in the crystal surface such that both components of the unit normal vector $\mathbf{n}$ are irrational. For this reason solitary corners lie in the gaps between different settings. To calculate the fractal dimension we must plot all the settings which have the longitudinal length $\Delta L(\mathbf{l})$ exceeding some small $\Delta$ (Fig. 1). Since $\Delta L(\mathbf{l})$ is given by equation (1), we must consider all the planes given by the equations $\mathbf{n} . \mathbf{l}=0$ where $|\mathbf{l}|<\Lambda ;\left(\Delta=\zeta(\gamma-1) / \Lambda^{\gamma-1}\right)$. Each plane of this form brings about a one-parameter subset on the crystal surface. The number of these settings is equal to the number of integer vectors 1 shorter than $\Lambda$, which is [7] :

$$
K \approx \frac{4}{3} \pi \frac{1}{\zeta(3)} \Lambda^{3}
$$

The crystal surface consists of $K$ largest settings and of $M$ gaps. Every gap is a region on the crystal surfaces which size in both directions is of the order of $\Delta$ (Fig. 1). The number of the gaps can be found by means of the Euler theorem (the number of facets plus the number of corners minus the number of edges equals 2 ) and of number theory : $M \sim K^{2} \sim \Lambda^{6}$. Comparing this formula with $\Delta \sim 1 / \Lambda^{\gamma-1}$ we have

$$
M \sim \Delta^{-d} \quad d=\frac{6}{\gamma-1}
$$

that yields the fractal dimension.

\section{Experimental predictions.}

The power laws (3), (5) or (7) are valid at sufficiently large index $N=\sqrt{p^{2}+q^{2}+r^{2}}$. Consider the question : which value of $N$ can be considered large ? This minimal $N$ depends on two factors. 
One is the form of $U(\mathbf{r})$ at small $r$, another is associated with number theory, in particular, with formulae (2) and (6). The most favourable setting for experimental investigation is an « equatorial " setting (shaded in Fig. 1). It consists of $\{p, q, 0\}$ facets and of solitary edges parallel to the $Z$ axis. To simulate an experimental measurement we have substituted $U(\mathbf{r})=-1 / r^{6}$ into theoretically obtained formulae of I and calculated numerically the widths of all $\{p, q, 0\}$ facets larger than some scale $\Delta$ and the widths of gaps between them. It is seen from figure 2a that if we suppose that $U(\mathbf{r})=-1 / r^{\gamma}$ for any $r$, the power law (5) holds for $N \gtrsim 3$. Thus if we assume that the Van der Waals attraction dominates at $r \gtrsim 5$ then the law (5) is valid for the chosen setting for $N \gtrsim 5$. Figure 2 shows that if an experimentalist can keep the crystal in equilibrium in such a

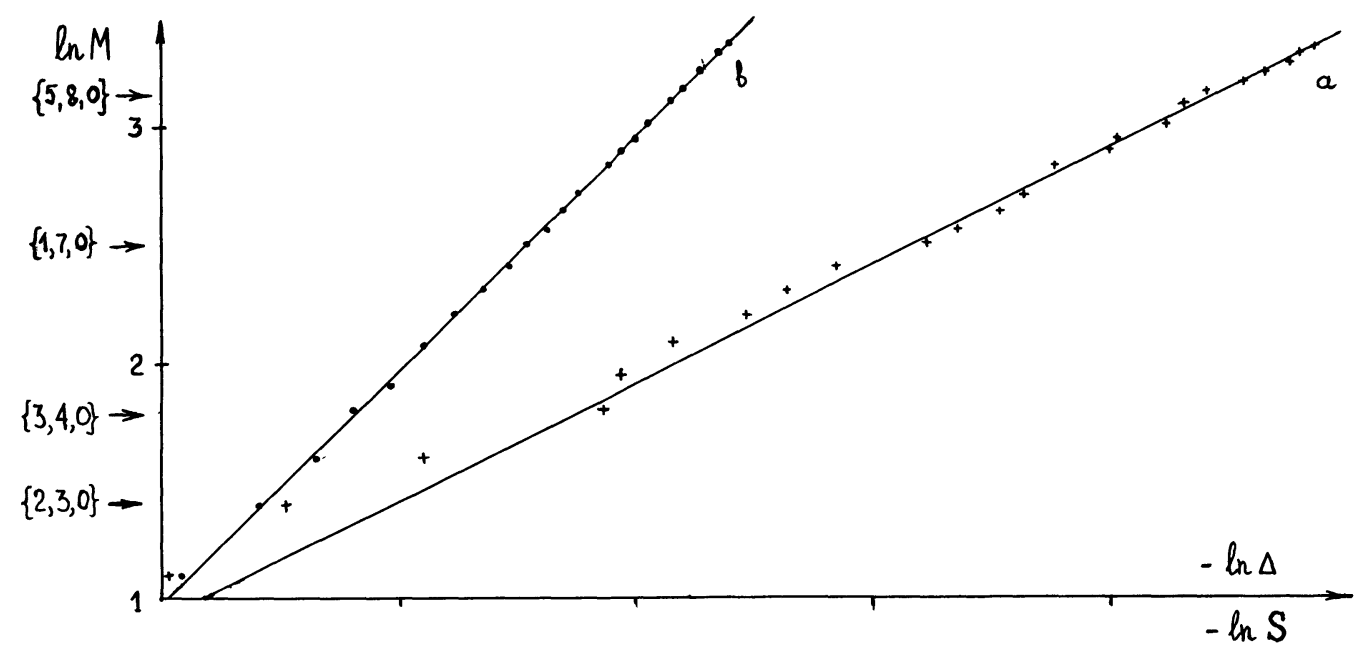

Fig. 2. - Number of discernible facets $M$ as a function of a scale $\Delta$ (a), and of a total width of all gaps between discernible facets $S(b)$. Miller indices of the smallest discernible facets are shown. The slopes are consistent with equations (5) and (8).

way that the facet $\{1,7,0\}$ is discernible, the fractal dimension of the Cantor set of the solitary edges can be measured. It should be noted that the function $\Delta(M)$ oscillates near the curve $\Delta=$ const. $M^{-1 / d}$ (Fig. 2a). There is an analogous function (Fig. 2b) with smaller oscillations. To obtain it one must consider the setting (Fig. 1) or its cross-section by the $x y$-plane (Fig. 6 of I) with the accuracy up to $\Delta$. Then one must measure the total width $S$ of all the gaps between the facets. Since $S \sim M . \Delta$ and $M \sim \Delta^{-\frac{2}{\gamma-2}}$, the function $S(M)$ is

$$
S \sim M^{-\left(\frac{\gamma-4}{2}\right)} \text {. }
$$

Equation (8) is equivalent to equation (5) at $M \rightarrow \infty$ but at finite $M$ may give a more accurate result than $\Delta(M)$ (Fig. 2).

When this Letter was in press the paper [8] considering faceting problem appeared.

\section{Acknowledgments.}

The author is thankful to K. M. Khanin and M. Yu. Rosenblum for useful discussions. 


\section{References}

[1] Keshishev, K. O., Parshin, A. Yu., and Babkin, A. V., Sov. Phys. JeTP 53 (1981) 362.

[2] Wolf, P. E., Balibar, S. and Gallet, F., Phys. Rev. Lett. 51 (1983) 1366.

[3] Rottman, C., Wortis, M., Heyraud, J. C. and Metois, J. M., Phys. Rev. Lett. 52 (1984) 1009.

[4] Landau, L. D. and Lifshitz, E. M., Statistical Physics, Part I (Pergamon, Oxford) 1980, p. 520-521.

[5] Burkov, S. E., J. Physique 46 (1985) 317.

[6] Landau, L. D., Collected papers translated by D. Ter Haar (Gordon and Breach, New York) 1965, p. $540-545$.

[7] VinoGradov, I. M., Foundations of Number Theory (Nauka, Moscow) 1972 (Russian).

[8] Schulz, H. L., J. Physique 46 (1985) 257. 